\title{
A Method for Differentially Coherent Detection of OFDM Signals on Doppler-Distorted Channels
}

\author{
Milica Stojanovic \\ Northeastern University \\ millitsa@ece.neu.edu
}

\begin{abstract}
Doppler distortion causes inter-carrier interference which prevents the use of differentially coherent detection in OFDM systems. To recover this efficient detection method, we propose to use several FFT demodulators operating in parallel over non-overlapping time segments, and to combine their outputs prior to detection. This technique aims for efficient implementation of front-end matched filtering, followed by differential MMSE combining. A low complexity algorithm is proposed for recursive computation of combiner weights, and its gain over the conventional detector is quantified through numerical examples of an underwater acoustic channel with severe Doppler distortion.
\end{abstract}

\section{INTRODUCTION}

Motivated by application to underwater acoustic (UWA) communications, we investigate the use of differentially coherent detection in conjunction with orthogonal frequency division multiplexing (OFDM). OFDM is used in a variety of radio systems (wireless LAN, DAB/DVB, LTE), but has only recently been considered for UWA systems (see e.g. [1] and references therein). OFDM offers simplicity in dealing with the frequency selectivity, which can be fairly severe in an acoustic channel (a multipath spread $T_{m} \sim 10 \mathrm{~ms}$ is commonly observed in shallow water systems operating within bandwidths $B \sim 10 \mathrm{kHz}$ ).

Differentially coherent detection eliminates the need for channel estimation, relying instead on the assumption that the channel response changes slowly, either between carriers (differential encoding/detection in frequency) or between blocks (differential encoding/detection in time). If the channel is perfectly known at the receiver, differential detection incurs a penalty (the exact amount depends on the fading); however, channel estimation errors degrade the performance of coherent detection, rendering it equal or even inferior when the channel variation is non-negligible [2]. Moreover, differential detection eliminates the overhead of pilot-assisted channel estimation.

We consider here differential encoding/detection in frequency, as it plays hand-in-hand with the system design that targets efficient use of bandwidth. Bandwidth efficiency is given by the ratio of the symbol rate to bandwidth, $R / B \sim$ $1 /\left(1+T_{m} B / K\right)$, where $K$ is the number of carriers. Hence, to maximize the bandwidth efficiency, the greatest possible number of carriers should be used. Increasing the number of carriers in a fixed bandwidth (presumably limited for an acoustic system) will result in narrowing the subband width $\Delta f=$ $B / K$, favoring the use of differential encoding/detection in frequency, as it simultaneously supports increased coherence between adjacent carriers and increased bandwidth efficiency.
The number of carrier, however, cannot grow beyond the time-coherence limit of an OFDM system. As the block duration $T=1 / \Delta f$ increases, motion-induced frequency offset as well as inherent variation in the path gains can no longer be ignored, and the channel no longer appears fixed over one block. Orthogonality of the received carriers is thus lost, and inter-carrier interference (ICI) arises. Simple differentially coherent detection is no longer justified, while coherent detection has to resort to ICI equalization. ICI equalization techniques include basic methods (see e.g. [3]) as well as those based on explicit modeling of the channel's time-variation as piece-wise linear (e.g. [4]). ICI equalization in UWA systems has been addressed in [5].

The use of differentially coherent OFDM over channels with non-negligible time-variation was addressed in [2], where ICI self-cancellation [6] was coupled with differential encoding in frequency. Self-cancellation is a form of transmitter precoding, developed for channels whose time variability comes from carrier frequency offset. ICI on such channels exhibits symmetry, which is exploited by arranging the data symbols onto carriers so that the resulting interference cancels itself. Specifically, the same data symbol can be used to modulate a pair of adjacent carriers (with opposite sign) [6], or a pair of non-adjacent carriers [7]. However, ICI self-cancellation comes at the price of reduced data rate.

While the various approaches considered in the literature focus on post-FFT signal processing, in this paper we take a different approach which aims to exploit the information about the channel's time-variation before it has been lost in the process of demodulation. The proposed demodulator operates on partial, non-overlapping segments of an OFDM block, which are later combined. Effectively, it aims to perform adaptive matched filtering, i.e. to implement the receiver frontend in an optimal manner [8]. Several FFTs are now needed instead of one, but this is a small increase in computational complexity.

After combining the partial FFT demodulator outputs, the residual ICI is not eliminated, but it is expected to be sufficiently reduced so that differentially coherent detection can follow. The receiver's only non-trivial task is to determine the combiner weights. We propose an efficient algorithm that computes the combiner weights recursively across the carriers. The algorithm is based on minimizing the mean squared error (MSE) in the estimated data sequence after differential combining, and it uses some of the ideas presented in [9]. 
The paper is organized as follows. In Sec.II we outline the principle of partial FFT demodulation. Sec.III presents the algorithm for determining the combiner weights. System performance is illustrated in Sec.IV using a simulated example of an underwater acoustic channel. Finally, conclusions are summarized in Sec.V.

\section{System Model and Partial FFT Demodulation}

The received signal is modeled as

$$
r(t)=\sum_{p=0}^{P-1} h_{p}(t) s\left(t-\tau_{p}(t)\right)+n(t)
$$

where $h_{p}(t)$ is the (real-valued) path gain corresponding to the $p$-th (physical) propagation path, $\tau_{p}(t)$ is the path delay whose time-variation we will model as $\tau_{p}(t)=\tau_{p}-a_{p} t$, where $a_{p}$ can be positive or negative (compression or dilation); $n(t)$ is the noise, and

$$
s(t)=\operatorname{Re}\left\{\sum_{k=0}^{K-1} d_{k} e^{j 2 \pi f_{k} t}\right\}, \quad t \in\left(-T_{g l}, T+T_{g r}\right]
$$

is the transmitted signal containing $K$ data symbols $d_{k}$ modulated onto the carriers of frequency $f_{k}=f_{0}+k \Delta f$. The basic OFDM block duration $T=1 / \Delta f$ is extended by the cyclic prefix of length $T_{g}=T_{g l}+T_{g r}$, long enough to capture the effects of multipath and Doppler spreading. The data symbols $d_{k}$ are obtained by differential encoding of the original i.i.d. symbols $b_{k}$, i.e. $d_{k}=d_{k-1} b_{k}$, where $b_{k}$ and $d_{k}$ assume values from the same PSK alphabet, with $d_{0}=1$. The symbol rate is $R=K /\left(T+T_{g}\right)=B /\left(1+T_{g} B / K\right)$.

The principle of partial FFT demodulation is illustrated in Fig.1. The input signal $v(t)$ is the received signal $r(t)$ down-converted by the lowest carrier frequency $f_{0}$. While the conventional demodulator produces one output $y_{k}$ per carrier, partial demodulator yields $M$ outputs per carrier,

$$
y_{k, m}=\int_{(m-1) T / M}^{m T / M} v(t) e^{-j 2 \pi k \Delta f t} d t, \quad m=1, \ldots M
$$

The $M$ outputs are then combined to yield

$$
y_{k}=\sum_{m=1}^{M} p_{k, m}^{*} y_{k, m}=\mathbf{p}_{k}^{\prime} \mathbf{y}_{k}
$$

where the prime denotes conjugate transpose. Conventional demodulation can thus be regarded as a special case of partial demodulation in which $p_{k, m}=1$. This choice provides ideal (orthogonal) detection variables on a time-invariant channel. When the channel is time-varying, a judicious selection of the combiner weights $\mathbf{p}_{k}^{\prime}$ for each carrier allows additional freedom in suppressing the ICI. We note a resemblance to array processing.

In practice, OFDM demodulation is performed using FFT instead of continuous-time integration. FFT can also be used to perform partial demodulation, as illustrated in Fig.2. Each FFT block is of the same size as the one used at the transmitter (or in a conventional demodulator), but it operates on a vector of windowed signal samples. For example, the second FFT in Fig.2 operates on the received signal vector in which only the samples corresponding to $(T / 4, T / 2)$ are kept, while all the others are set to zero.
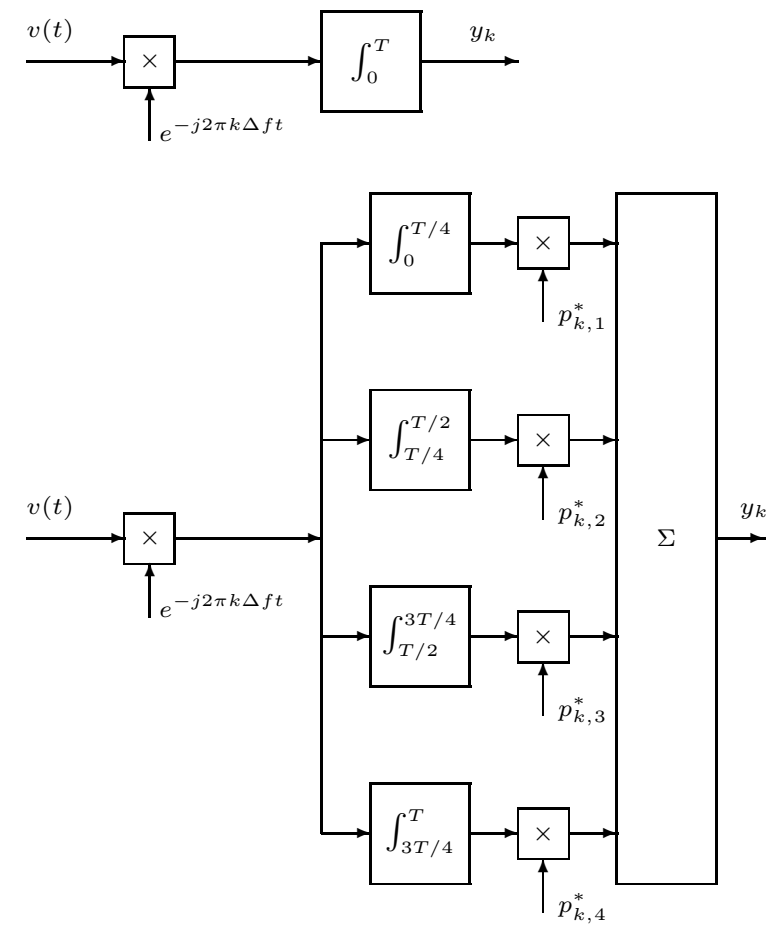

Fig. 1. Demodulation on the $k$-th carrier: Conventional (top) and partial demodulation with $M=4$ intervals (bottom). Partial demodulation allows each time segment to be weighted before combining.

\section{Differentially Coherent Detection}

Differentially coherent detection is based on the assumption that the demodulator output can be modeled as

$$
y_{k}=H_{k} d_{k}+z_{k}
$$

where $H_{k}$ represents the channel distortion which changes slowly across the carriers, and $z_{k}$ contains the noise (and small amounts of noise-like residual ICI). Differential combining then yields the decision variable $\hat{b}_{k}=y_{k} / y_{k-1}$, which can be used to make decisions $\tilde{b}_{k}$ on the transmitted data symbols.

Using the expression (4), we have that

$$
\hat{b}_{k}=\frac{\mathbf{p}_{k}^{\prime} \mathbf{y}_{k}}{\mathbf{p}_{k-1}^{\prime} \mathbf{y}_{k-1}}
$$

We now want to determine the combiner weights so as to minimize the MSE, $E\left\{\left|e_{k}\right|^{2}\right\}$, where

$$
e_{k}=\hat{b}_{k}-b_{k}
$$

Minimization is complicated by the fact that $\mathbf{p}_{k}$ and $\mathbf{p}_{k-1}$ are not independent. The problem is similar in structure to that of differentially coherent equalization, where it is usually avoided by ignoring the dependence to arrive at the Wiener solution [9]. An alternative approach, and one that is better justified in the 


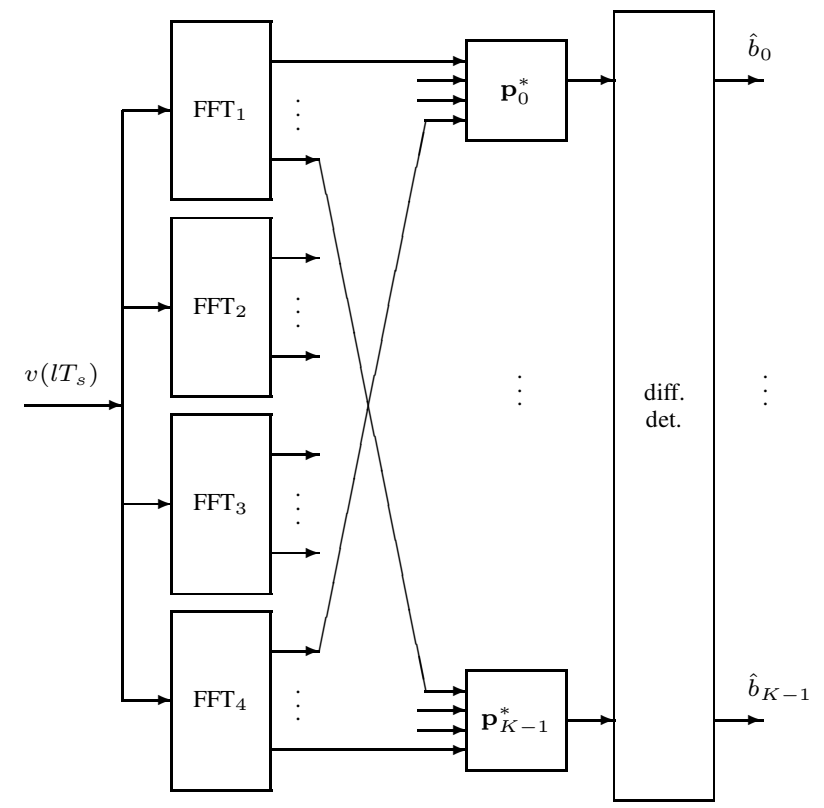

Fig. 2. Differentially coherent detection with partial FFT demodulation. FFT $_{1}$ operates on the first partial interval $(0, T / 4), \mathrm{FFT}_{2}$ on the second, etc. After combining, the signals are passed to the differential detector. The combiner weights $\mathbf{p}_{k}^{*}$ are computed recursively across the carriers to minimize the MSE in the estimated data symbols $\hat{b}_{k}$.

present case, is to do precisely the opposite. Namely, since the carriers are assumed to be very closely spaced, we assume that the optimal values of $\mathbf{p}_{k}$ and $\mathbf{p}_{k-1}$ are close too, i.e. that $\mathbf{p}_{k-1}^{\prime} \mathbf{y}_{k-1} \approx \mathbf{p}_{k}^{\prime} \mathbf{y}_{k-1}$. With this assumption, the squared error gradient is found to be

$$
\mathbf{g}_{k}=\frac{\partial\left\{\left|e_{k}\right|^{2}\right\}}{\partial \mathbf{p}_{k}^{*}}=\frac{1}{y_{k-1}^{2}}\left[\mathbf{y}_{k} \cdot y_{k-1}-y_{k} \cdot \mathbf{y}_{k-1}\right] e_{k}^{*}
$$

A stochastic gradient algorithm with a step size $\mu$ can now be applied to solve for the MMSE combiner weights recursively:

$$
\mathbf{p}_{k+1}=\mathbf{p}_{k}+\mu \mathbf{g}_{k}
$$

The algorithm operates in a decision-directed manner, using $\tilde{b}_{k}$ instead of the true values $b_{k}$ to compute the error needed for the gradient (8). Since the combiner vectors are short (e.g. $M=2$ or 4) the training overhead is small. Additional pilots can be inserted periodically, to guard against error propagation.

\section{NUMERICAL EXAMPLES}

In this section, we illustrate the algorithm performance using a numerical example. Fig. 3 shows the nominal characteristics of our test channel. The path gains are calculated from the channel geometry, using the acoustic propagation loss for the given frequency range, ${ }^{1}$ and assuming ideal plane wave propagation with surface reflection coefficient -1 , and each bottom reflection coefficient calculated according to the

\footnotetext{
${ }^{1}$ The acoustic path loss over distance $l_{p}$ is modeled as $A\left(l_{p}, f\right)=$ $A_{0} l_{p}^{\kappa} \mathrm{a}(f)^{l_{p}}$, where $A_{0}$ is a constant, $\kappa$ is the spreading factor, and a $(f)$ is the frequency-dependent absorption coefficient [10].
}

incident angle [11]. The frequency response is highly selective, with coherence frequency $1 / T_{m} \sim 400 \mathrm{~Hz}$.

Time variation is modeled through the Doppler factor $a$, taken equal for all the propagation paths. This simple model is used for purposes of illustration, while more elaborate models are left for a later study.
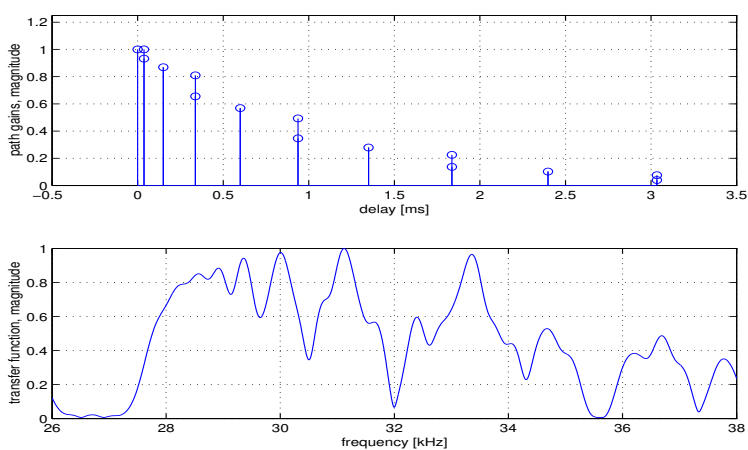

Fig. 3. Underwater acoustic test channel: nominal characteristics for $2 \mathrm{~km}$ distance, $15 \mathrm{~m}$ water depth, transmitter and receiver in mid-water, soft bottom (sound speed $1400 \mathrm{~m} / \mathrm{s}$, density $1800 \mathrm{~g} / \mathrm{m}^{3}$ ) and spreading factor $\kappa=1.5$.

The algorithm performance is summarized in Fig.4. This figure shows the MSE (average over carriers, simulation runs) as a function of the Doppler factor, which ranges between $10^{-6}$ and $10^{-4}$. These values of the Doppler factor should be thought of as residuals after initial re-sampling. The actual time scaling can occur at an even higher rate in a mobile acoustic channel, but this is normally controlled by (coarse) initial synchronization. The noise $n(t)$ is modeled as zeromean white Gaussian, ${ }^{2}$ with power spectral density $N_{0} / 2$. The MSE is used as a figure of merit for the performance of signal processing (the ultimate BER will also depend on the channel code). The results of Fig.4 were obtained for an uncoded system, with the bit SNR set to $E_{b} / N_{0}=30 \mathrm{~dB}$. The results scale for different values of the SNR, but the general trends remain the same.

Fig.4 clearly demonstrates the gain obtained by differentially coherent combining of partial FFT demodulator outputs. The MSE increases with the Doppler factor, eventually limiting the system performance, but it does so more slowly for the partial FFT combiner than for the conventional receiver. At $a=10^{-4}$, the $M=4$ scheme with $K=512$ carriers gains $10 \mathrm{~dB}$ over the conventional detector. A Doppler factor of $10^{-4}$ is extreme compared to what is typically found in radio systems, but it can easily occur in an UWA system, where relative motion on the order of a few $\mathrm{m} / \mathrm{s}$ is leveraged against the low speed of propagation $(1500 \mathrm{~m} / \mathrm{s})$.

The slower increase of MSE effectively results in extending the range of tolerable Doppler distortion. Namely, if the MSE has to be kept below a certain threshold, say $-5 \mathrm{~dB}$, in order to satisfy a BER requirement, this threshold translates into a maximum tolerable value of $a$, which is greater for $M=2$ and 4

${ }^{2}$ AWGN model is only an approximation for the acoustic noise, whose power spectral density decays with frequency (approximately linearly on the logarithmic scale) [10] 

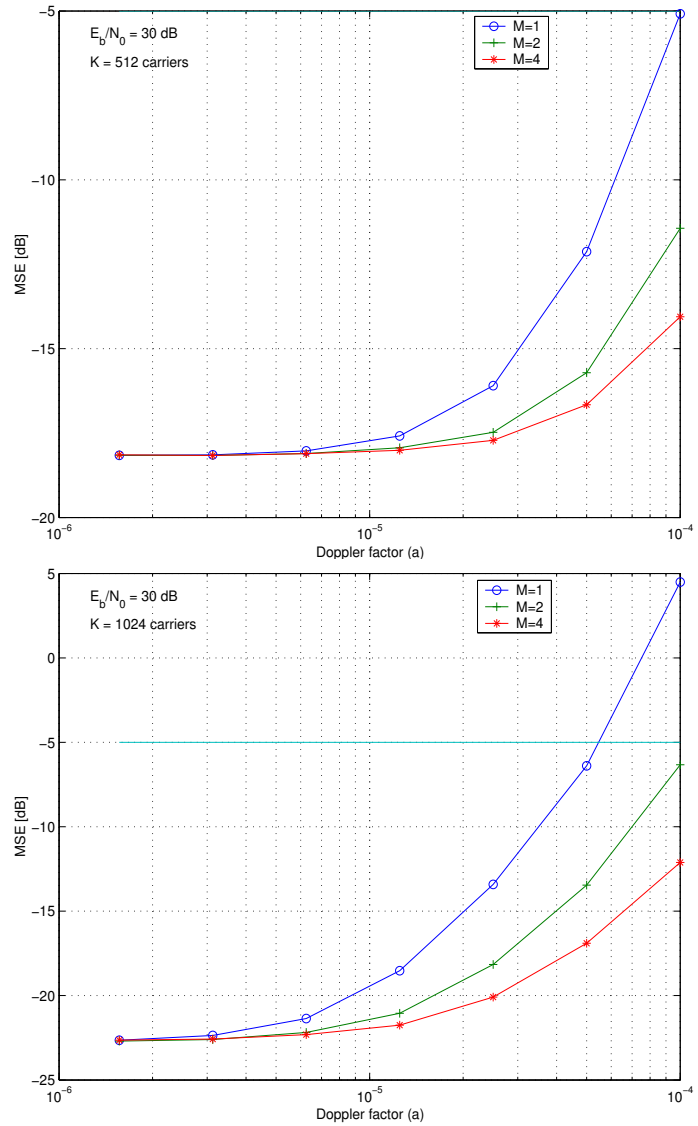

Fig. 4. Performance of differentially coherent detection with conventional demodulation $(M=1)$ and partial FFT demodulation $(M=2,4 ; \mu=0.01)$ Two cases are compared, OFDM with $K=512$ carriers (top) and $K=1024$ carriers (bottom).

than for the conventional system. Interesting implications arise when this fact is viewed in light of choosing the number of carriers. Fig. 4 compares two choices of $K, 512$ and 1024. In a conventional system with 1024 carriers, the MSE increases rapidly, exceeding the $-5 \mathrm{~dB}$ threshold at $a>5 \cdot 10^{-5}$. This is not surprising if one considers the underlying timevariation: at $a=10^{-4}$, the phase change experienced on the $k$-th carrier over the block duration $\left(2 \pi a f_{k} T\right)$ amounts to $2 \pi / 3$ at the high band edge ( $2 \pi / 5$ at the low band edge). If such values of the Doppler factor are to be expected, the conventional system has to be designed with fewer carriers. With $K=512$ (Fig.4, top) the $M=1 \mathrm{MSE}$ is kept below -5 $\mathrm{dB}$ for all $a<10^{-4}$. The price for reducing the number of carriers is not only in decreased bandwidth efficiency, but also in decreased performance at lower values of $a$. (The system is designed to tolerate some maximal Doppler distortion, but the actual value changes slowly with motion and can be lower.) Since the carriers are farther apart $\left(\Delta f \approx 1 / 16 T_{m}\right.$ at $K=512$ as opposed to $1 / 32 T_{m}$ at $K=1024$ ) differentially coherent detection is more challenging. Instead of reducing the number of carriers, the situation can easily be remedied by using partial FFT combining: with 1024 carriers and either 2 or 4 FFT demodulators, the MSE is kept below $-5 \mathrm{~dB}$ for all $a<10^{-4}$.
The combiner achieves this similarly as a phased array wouldby aligning the carrier phase on each partial segment before addition. Note, however, that the gradient algorithm is not constrained, but can be used to compensate for an arbitrary time-variation of the channel, including that of the path gains.

\section{Conclusions}

To enable differentially coherent detection on time-varying channels, a method was proposed in which several FFT demodulators operate over non-overlapping segments of an OFDM block to provide outputs which are then combined prior to differential detection. The combiner weights are designed to minimize the post-detection MSE, and are computed recursively (across the carriers for differential encoding/detection in frequency) using a stochastic gradient algorithm of linear complexity. System performance is illustrated through a numerical example of a shallow water channel with (non-uniform) Doppler distortion. The results clearly show the gain obtained by using only a few FFTs (2 or 4) with the matching number of combiner weights per carrier. Compared to conventional detection, this type of processing can simultaneously achieve improved performance and higher bandwidth efficiency. Future work will include addition of multiple receiving elements and experimentation with real acoustic data, as well as investigation of alternative algorithms for computing the combiner weights.

\section{ACKNOWLEDGMENT}

This work was supported by the ONR grants N00014-10-1-0576 and N00014-07-1-0738.

\section{REFERENCES}

[1] M.Stojanovic, "MIMO OFDM Over underwater acoustic channels," in Proc. 43rd Asilomar Conf. on Signals, Systems and Computers, Nov. 2009.

[2] S.Lu and N.Al-Dhahir, "Coherent and differential ICI cancellation for mobile OFDM with application to DVB-H," IEEE Trans. Wireless Commun., vol.7, No.11, pp. 4110-4116, Nov. 2008.

[3] L.Rugini, P.Banelli and G.Leus, "Low-complexity banded equalizers for OFDM systems in Doppler spread channels," EURASIP Journal on Applied Signal Processing, vol.2006, pp.1-13, April 2006.

[4] Y.Mostofi and D.Cox, "ICI mitigation for pilot-aided OFDM mobile systems," IEEE Trans. Wireless Commun., vol.4, No.2. pp.765-774, March 2005.

[5] K.Tu, D.Fentonati, T.Duman and P.Hursky, "Mitigation of intercarrier interference in OFDM systems over underwater acoustic channels," in Proc. IEEE Oceans Conf., May 2009.

[6] Y.Zhao and S.G.Haggman, "Intercarrier interference self-cancellation scheme for OFDM mobile communication systems," IEEE Trans. Commun., vol.49, No.7, pp. 1185-1191, July 2001.

[7] K.Sathananthan, R.Rajatheva and S.Slimane, "Cancellation technique to reduce intercarrier interference in OFDM," IEEE Electronic Letters, vol.36, No.25, pp.2078-2079, Dec. 2000.

[8] S.Yerramalli, M.Stojanove and U.Mitra, "Partial FFT demodulation: A detection method for Doppler distorted OFDM Systems," in Proc. Int'l Workshop on Sig. Proc. Advances in Wireless Commun. (SPAWC), 2010.

[9] M.Stojanovic, "An adaptive algorithm for differentially coherent detection in the presence of intersymbol interference," IEEE J. Select. Areas Commun., vol.23, No.9, pp.1884-1890, Sept. 2005.

[10] M.Stojanovic, "On the relationship between capacity and distance in an underwater acoustic communication channel," ACM SIGMOBILE Mobile Computing and Communications Review (MC2R), vol.11, Issue 4, pp.34-43, Oct. 2007.

[11] F.Jensen, W.Kuperman, M.Porter and H.Schmidt, Computational Ocean Acoustics, Springer Verlag, 1994. 